\title{
LEPTON COLLIDER OPERATION WITH CONSTANT CURRENTS*
}

\author{
U. Wienands ${ }^{\dagger}$, SLAC, Stanford, CA, USA
}

\section{Abstract}

Electron-positron colliders have been operating in a topup-and-coast fashion with a cycle time depending on the beam life time, typically one or more hours. Each top-up involves ramping detector systems in addition to the actual filling time. The loss in accumulated luminosity may be 20$50 \%$. During the last year, both B-Factories have commissioned a continuous-injection mode of operation in which beam is injected without ramping the detector, thus raising luminosity integration by always operating at peak luminosity. Constant beam currents also reduce thermal drift and trips caused by change in beam loading. To achieve this level of operation, special efforts were made to reduce the injection losses and also to implement gating procedures in the detectors, minimizing dead time. Beam collimation can reduce injection noise but also cause an increase in background rates. A challenge can be determining beam lifetime, important to maintain tuning of the beams.

\section{INTRODUCTION}

Both $B$-Factories, KEKB \& Belle in Tsukuba, Japan[1] and PEP-II \& BaBar at SLAC, USA[2], have adopted continuous injection (dubbed "trickle charge" at PEP) during their 2004 running. PEP-II, using the powerful SLAC linac as injector, injects several times a second into each of the rings quasi simultaneously. At KEKB, injection switches between $\mathrm{e}^{+}$and $\mathrm{e}^{-}$every $5 . .20 \mathrm{~min}$-with the detector ramped up-leading to some small variation of the beam current but the salient features of constant-current running are realized there as well.

An attempt to quantify the gains from trickle-charge into PEP is shown in Fig. 1, where the luminosity for one shift in normal running (bottom graph) is compared with constant-current operation for the LER only and for both rings. For LER-only, luminosity lifetime has already gone up by about $50 \%$, and the average length of a fill has significantly increased as well. The operating parameters for normal, LER-only and both-ring "trickle-charge" mode are compared in Table 1.

For KEKB, a plot comparable to the PEP evaluation is shown in Fig. 2. Table 2 summarizes the performance parameters (KEKB never operated with continuous injection into only one ring). Note that even though the beam currents are modulated by the $5 \ldots 20 \mathrm{~min}$. intervals in filling the electron and the positron ring, resp., there is little or no discernible modulation on the luminosity.

\footnotetext{
* Work supported by US DOE under contracts DE-AC02-76SF00515 and DE-AC03-76SF00098

$\dagger$ uli@slac.stanford.edu
}

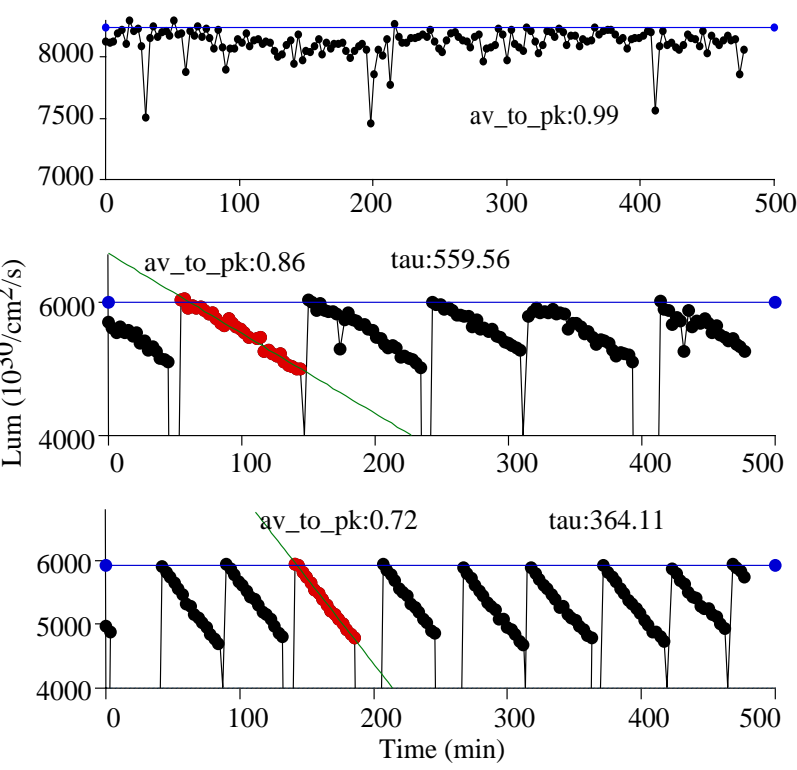

Figure 1: PEP luminosity LER trickling (top) and normal operation (bottom).

Table 1: PEP operational modes summary

\begin{tabular}{|l|l|l|l|}
\hline & Top-up & LER trkl & Both trickle \\
\hline Lum. lifetime & $364 \mathrm{~m}$ & $560 \mathrm{~m}$ & - \\
Avg./peak lum. & $72 \%$ & $86 \%$ & $99 \ldots 100 \%$ \\
Top-ups/shift & 10 & 6 & - \\
Lum. gain & - & $35 \%$ & $50 \%$ \\
\hline
\end{tabular}

While the direct gain from continuous-current operation is evident and easily quantified, there is also indirect gain arising from the increase in reliability due to less cycling of the facility. In case of PEP, trickle gain has exceeded the expected increase in delivered luminosity. This has been quantified and is indicated in Fig. 3.

A direct consequence of continuous-current operation is the higher average beam currents for the same peak current. Both facilities had to contend with the increase in heating arising from such increase and apply the appropriate re-

Table 2: KEKB operational modes summary

\begin{tabular}{|l|l|l|}
\hline & Top-up & Continuous injection \\
\hline Lum. lifetime & $280 \mathrm{~m}$ & - \\
Avg. to peak ratio & $75 \%$ & $99 \%$ \\
Top-ups/shift & 7 & - \\
Lum. Gain & - & $30 \%$ \\
\hline
\end{tabular}



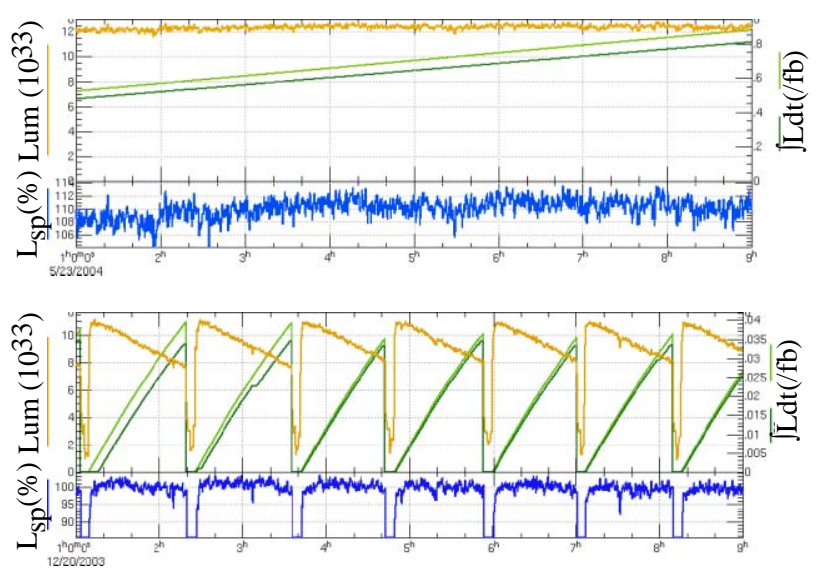

Figure 2: KEKB luminosity continuous injection (top) and normal operation (bottom).[3]

pairs.

Continuous injection with the detector ramped up requires tight cooperation between the machine and the detector groups. Both at KEKB as well as at PEP, the detector groups expended significant effort to modify their interlock systems to allow injection with ramped up systems as well as to provide background signals suitable for tuning of the injection quality.

\section{PEP-II}

Injection into the PEP rings is done in the vertical plane through a Lambertson septum, aligning the incoming beam with the ring in the horizontal plane, and a vertically bending current-sheet septum. The vertical $\beta$-function is high $(215 \mathrm{~m})$ to reduce the requirement for the septum and injection kickers. Two kicker magnets $180^{\circ}$ apart create a closed bump for the orbit; a 4-magnet dc bump allows to precisely align the incoming and circulating beam. Due to the SLC Damping Rings, the injected beam emittance

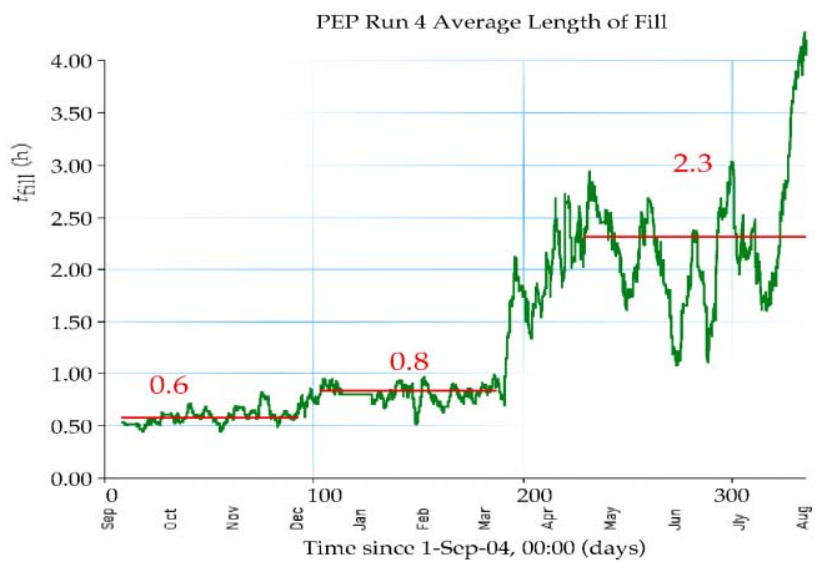

Figure 3: Average length of a fill for PEP-II in top-up-andcoast operation, LER-only trickle charge and both rings trickle charging. is smaller than the circulating-beam emittance. Table 3 summarizes the parameters. Continuous injection (trickle-

Table 3: PEP-II injection parameters[4]

\begin{tabular}{|l|c|c|}
\hline Parameter & $\mathbf{e}^{+}$ & $\mathbf{e}^{-}$ \\
\hline Energy $(\mathrm{GeV})$ & $3.1 \mathrm{GeV}$ & $9 \mathrm{GeV}$ \\
$1-\sigma$ Emittance $(\mathrm{x} / \mathrm{y})$ & $6.6 / 0.8 \mathrm{nmr}$ & $2.3 / 0.3 \mathrm{nmr}$ \\
FWHM energy spread & $0.7 \%$ & $0.7 \%$ \\
Energy acceptance & $0.7 \%$ & $0.7 \%$ \\
$1-\sigma$ pulse length & $1 \mathrm{~mm}$ & $1 \mathrm{~mm}$ \\
\hline
\end{tabular}

charge) was first established in the PEPII Low Energy Ring (LER) where the stored-beam lifetime is lowest, and where injection background has been less of a problem than in the High Energy Ring (HER). Large vertical beta functions outside the detector in the HER case combined with vertical injection necessitate better control of incoming beam parameters. In the LER, the large vertical beta functions occur in the detector closer to the interaction point such that sensitivity to incoming beam parameters is greatly reduced. Several factors contributed to the success of trickle-charge operation:

- Background signals provided by the BaBar detector gated on actual injection pulses.

- Systematic improvements of the electron beam from the SLAC linac.

- Reduction of the distance of the injected beam from the closed orbit in the vertical plane.

- Trajectory stabilization feedback at injection.

- Both ring kicker systems were evaluated and have recently been upgraded to improve the match between the two kickers.[5]

The gated background signals proved to be a most effective tuning tool. The regular BaBar background detectors are insufficient for trickle-charge tuning as they do not discriminate between stored and injected background. A special diagnostic system counts BaBar calorimeter (EMC) triggers following an injection pulse, providing an injection-rate-independent background signal suitable for tuning. The EMC remains on even during normal injection (detector ramped down) thus allowing to use of this tool before trickle injection is activated.

Figure 4 (top) shows an example histogram of triggers $v s$ time since injection, for moderately well tuned injection. Bad conditions often show up as a bump in this histogram. An extension to this tool is an FFT plot of the time-sinceinjection of the injection triggers; this has proven to convey information about the matching of the injector energy to the ring. The bottom part of Figure 4 shows such spectrum with a peak at the synchrotron frequency of the HER, indicating an energy offset of the incoming beam.

Continuous injection created a desire to improve the quality of the match of the two injection kickers used to displace the closed orbit towards the septum during an injection pulse. The original system created a global orbit 

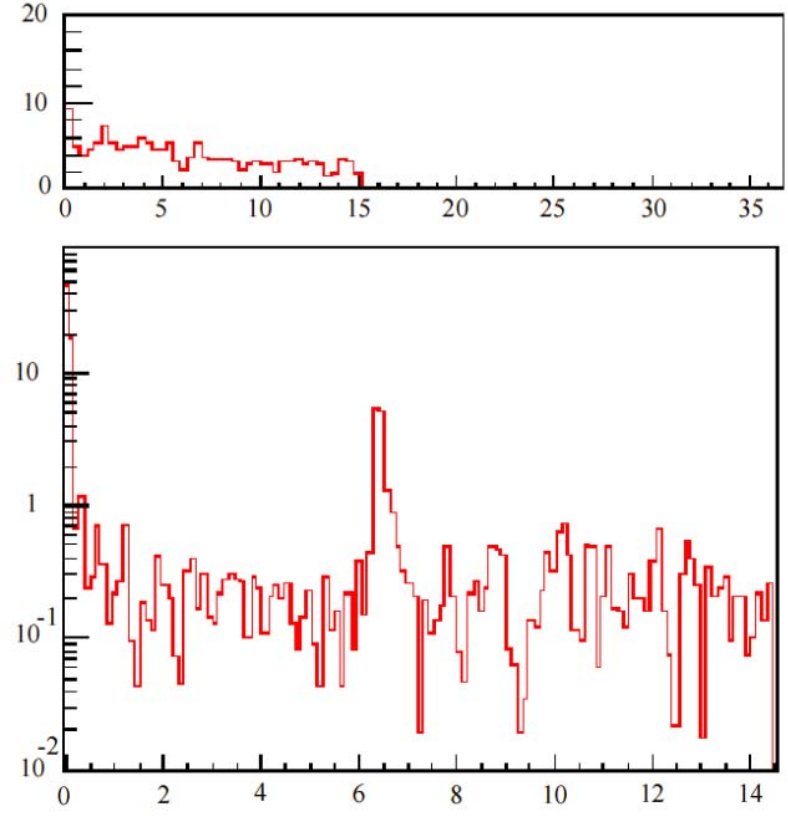

Figure 4: EMC triggers vs time from injection (top) and FFT of the triggers (bottom).

wave of about $0.5 \mathrm{~mm}$ peak around the ring (a few $\mu \mathrm{m}$ at the IP), clearly visible in luminosity dips on the fast luminosity monitor when the injection kicker fires. Recently, the modulators for both injection systems were upgraded to individual units for each magnet thus allowing accurate amplitude control and timing control. In this way the kicker mismatch has been reduced to about $10 \%$ of its former value and the effect of injection on luminosity is no longer significant.[6, 7]

The 15-ms duration of the injection background would require too long a window to be applicable without undue loss of event rate. However, since the detector trigger system can resolve a time scale less than $1 \mu \mathrm{sec}$ it is possible to use a periodic gate synchronized about the injecting bunch

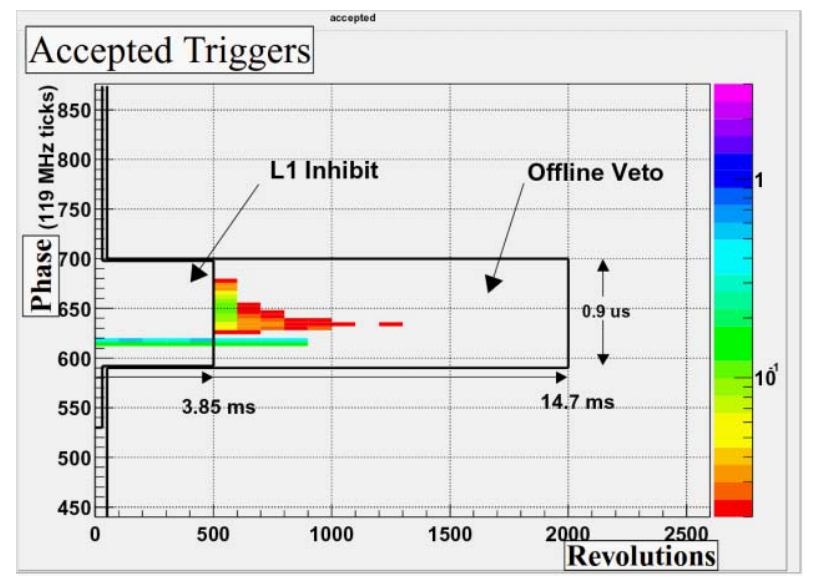

Figure 5: 2-d gating of the BaBar detector. with only $\pm 0.5 \mu \mathrm{sec}$ length for the first $15 \mathrm{~ms}$ after injection, thereby reducing the downtime to $<2 \%$. Fig. 5 shows a 2-d graphical representation of this gate.

In commissioning HER trickle-charge, the most severe issue have been high-background injection pulses ("fliers"). Linking BaBar's signals into the PEPII control system, we have been able to correlate incoming beam parameters with such pulses. While injected beam energy, phase (timing) and transverse position are measured and stabilized by feedback, jitter from faulty hardware or mis-set parameters is still possible. In particular, sourceintensity jitter can cause parameter changes of the injected beam. Such jitter has been reduced by progressive tuning and hardware repair. In Figure 6 the reduction of intensity jitter by tuning and repair is shown.[8]

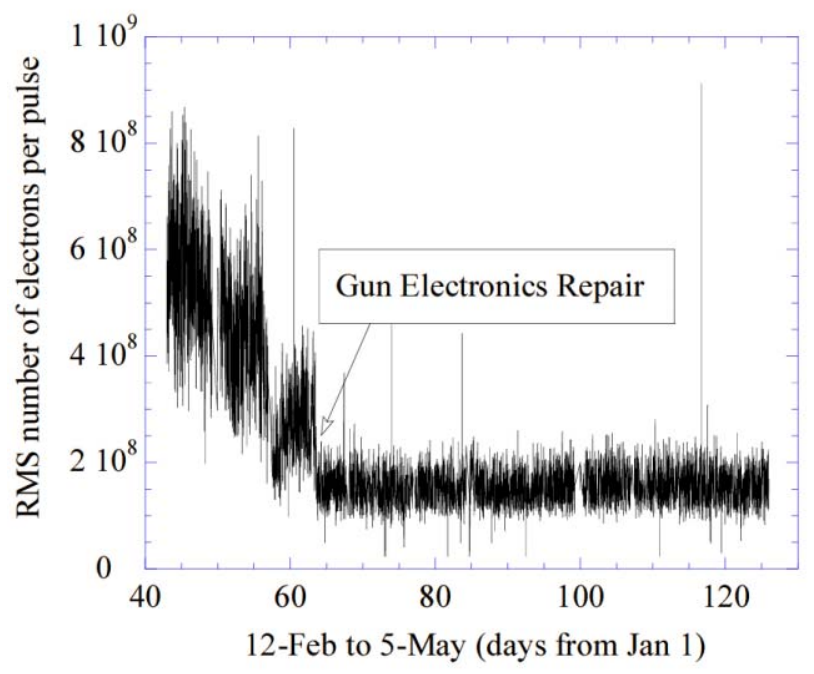

Figure 6: Reduction of electron intensity jitter by tuning and repair.

\section{KEKB}

The KEKB rings are injected in the horizontal plane. The beam parameters are summarized in Table 4 As there

Table 4: KEKB injection parameters[9]

\begin{tabular}{|l|c|c|}
\hline Parameter & $\mathbf{e}^{+}$ & $\mathbf{e}^{-}$ \\
\hline Energy $(\mathrm{GeV})$ & $3.5 \mathrm{GeV}$ & $8.5 \mathrm{GeV}$ \\
$1-\sigma$ Emittance & $220 \mathrm{nmr}$ & $16 \mathrm{nmr}$ \\
FWHM energy spread & $0.6 \%$ & $0.3 \%$ \\
Energy acceptance & $0.5 \%$ & $0.5 \%$ \\
$1-\sigma$ pulse length & $1.5 \mathrm{~mm}$ & $1.5 \ldots 3 \mathrm{~mm}$ \\
\hline
\end{tabular}

are no damping rings, the transverse emittance of the injected beam is larger than the circulating emittance. Also, the injector does not accelerate electrons and positrons at the same time, rather, injection is switched back and forth between these. For top-up-and-fill operation, KEKB 


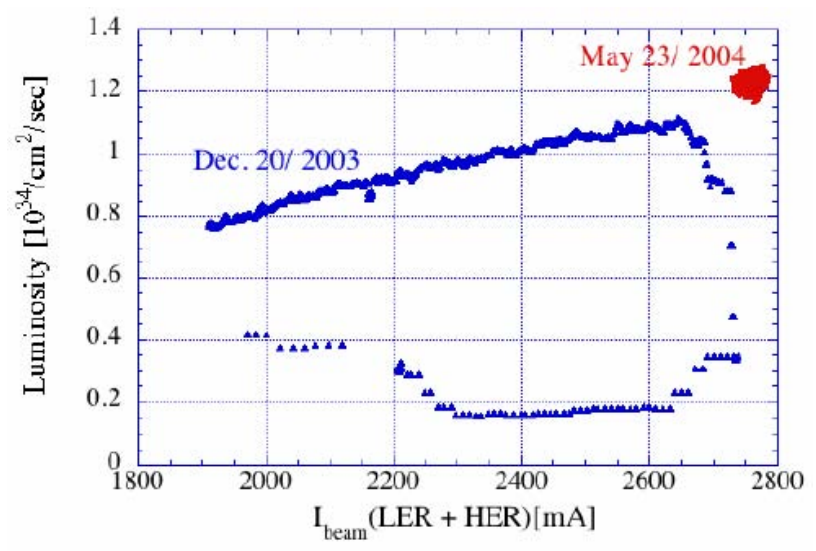

Figure 7: KEKB luminosity before and after continuous injection was established.[10]

would inject at $50 \mathrm{~Hz}$ repetition rate of the injector; this is lowered to $10 \mathrm{~Hz}$ for continuous injection. The intensity of the injector beam is maintained. Since the KEKB injector linac does not support simultaneous acceleration of positrons and electrons, the particle species is switched every $5 \ldots 20 \mathrm{~min}$. With typical beam lifetimes of $150 \ldots 200 \mathrm{~min}$., this is not a significant impediment. KEKB quotes an increase in integrated luminosity per shift of about $30 \%$, compared to top-up-and-fill operation, a few $\%$ of that arises from luminosity tuning happening at peak and constant beam current as opposed to shortly after injection stopped. The development of the specific luminosity supports this assertion, see Fig. 7 (a part of this increase is attributable to lowering $\beta_{y}^{*}$ ). This increase is only slightly offset by an about $2 \%$ increase in dead time due to the injection-inhibit gate of the Belle detector.

An injection-related background signal from the timeof-flight (TOF) system of the Belle detector is provided to

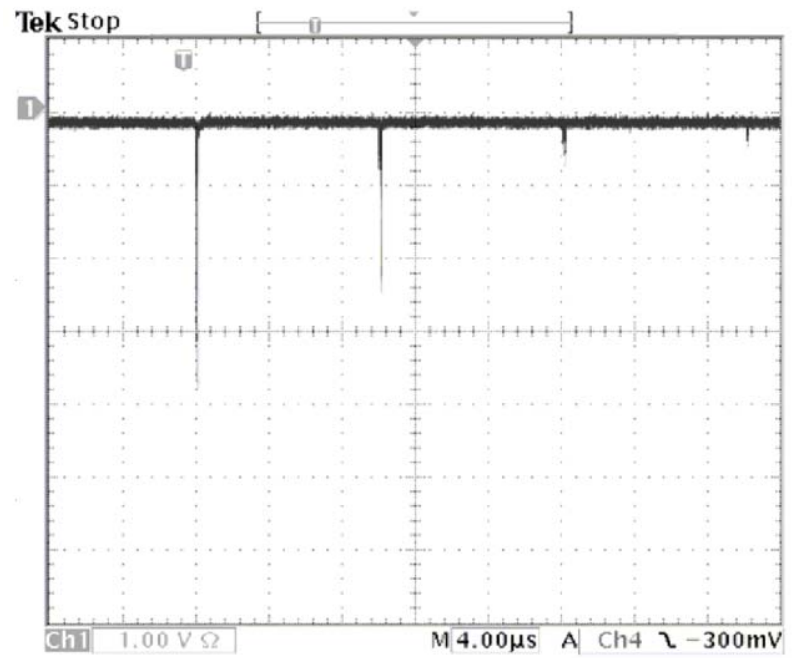

Figure 8: Belle L1 triggers during injection. Each vertical line is one injection pulse.[11] the machine operators to allow for tuning to minimize injection background. An example shown in Fig. 8 shows the turn-by-turn background after injection, while the same on a longer time scale shows the extent of the injection background over a longer time (Fig. 9). Comparing this figure to Fig. 4 it is evident that the background at KEKB has much shorter duration than that at PEP.

Like BaBar, the Belle detector needs to be gated to veto background events arising from the injected pulse. In case of Belle, however, the gating window is only $3.5 \mathrm{~ms}$ long, reflecting the shorter duration of the injection background. No gating on the bunch-level is taking place, i.e. the detector is insensitive for the whole duration of the inhibit gate. The increase in dead time from this gating is 3.5\% for $10 \mathrm{~Hz}$ injection rate; in practise the effective injection rate is closer to $2 / 3$ of $10 \mathrm{~Hz}$ and the additional dead time just above 2\%. The gating window is indicated in Fig. 9, upper trace.

A significant problem in Belle was uncovered during early tests of continuous injection: the preamplifiers for the TOF particle identifier system were not able to withstand the large-charge pulses arising from the injection losses and would lock up for a certain time after injection. Initially, the time constants of the amplifiers were shortened but eventually the problem was resolved by replacing all the amplifiers.[11]

Important parameters for smooth injection into KEKB are the collision conditions (incl. LER tunes), beam collimation and the ring acceptance (i.e. sextupole settings). While the parameters of the linac beam are obviously important, they are said to be stable. This differs from the experience at PEP, where the energy spread of the injecting beam is one of the most sensitive parameters, which is controlled by feedback but can occasionally become unstable and is also somewhat intensity dependent. KEKB also relies rather heavily on beam collimation; for continuous

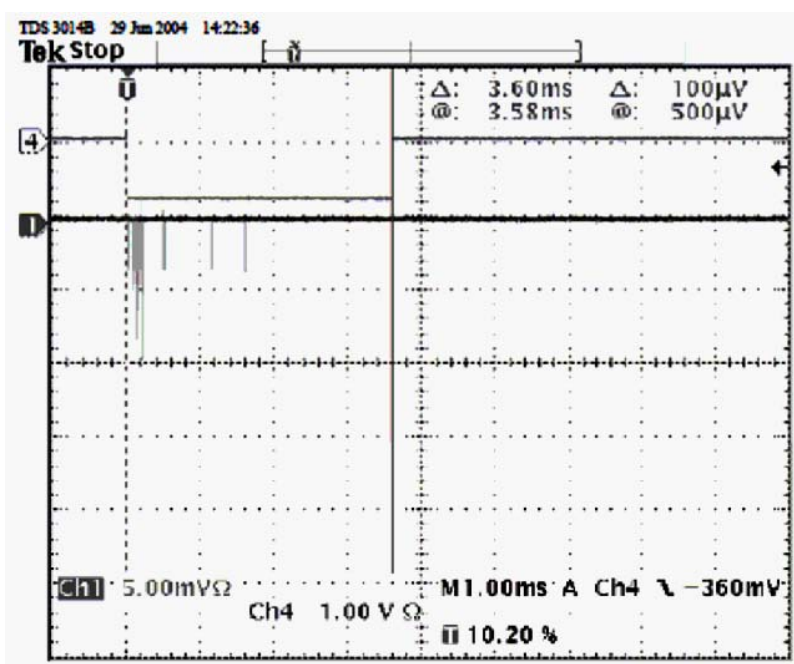

Figure 9: Same as Fig. 8 but $1 \mathrm{~ms} / \mathrm{div}$. time scale. The upper trace indicates the detector blank-out pulse.[11] 
Table 5: Summary of continuous-current operation

\begin{tabular}{|l|l|l|}
\hline Parameter & KEKB & PEP-II \\
\hline Injection plane & horiz. & vertic. \\
Beam lifetime & $250 / 200 \mathrm{~m}$ & $400 / 60 \mathrm{~m}$ \\
Gain & $30 \%$ & $30 \ldots 50 \%$ \\
Detector gate & $3.5 \mathrm{~ms}$ & $15 \mathrm{~ms} / 0.9 \mu \mathrm{s}$ \\
Deadtime due to gating & $3.5 \% *$ & $1.8 \%{ }^{*}$ \\
Average length of fill & $6 \ldots 8 \mathrm{~h}$ & $2.5 \ldots 4 \mathrm{~h}$ \\
Background reduction by collimation & $>2$ & $\approx 1.5$ \\
most important collimation & vertic. & horiz. \\
Background monitoring & Injection-gated from detector & dto. \\
Injection control & reduce rate & reduce rate and charge/pulse \\
\hline
\end{tabular}

* at $10 \mathrm{~Hz}$ injection rate

injection the vertical beam collimation is the most important. Detailed studies have not been done, but collimation is reducing background by at leas a factor of two, Interestingly, like at PEP this is the non-injecting plane.

\section{COMPARISON}

Reviewing the parameters in Table 5 the most significant difference appears to be the larger and longer-lasting background in PEP, dealt with by the fast 2-d gating in the BaBar detector such that the injection dead times for both facilities are comparable. The gain from continuouscurrent operation is similar for both facilities, although the increase in length of fill is greater in PEP, therefore the greater gain. About $5 \%$ of the gain for KEKB/Belle is attributed to tuning the machine at the highest beam currents. Beam collimation appears to be somewhat more important at KEK than at PEP: at KEKB, improperly setting of collimators can lead to almost $100 \%$ dead time. At PEP, while collimation is important and routinely used, its main effect is to keep the current in the Drift Chamber within acceptable limits and prevent the occasional bad linac pulse for causing difficulty.

An important operational aspect of continuous injection is beam-lifetime monitoring. Other than during top-upand-coast operation, short beam lifetime is not immediately evident except by producing high background in the detector, which can occur for different reasons. Presently, at both facilities the beam lifetime is monitored via the injection rate as shorter lifetime requires more frequent injections, with suspension of injection to get a direct measurement when conditions seem to be unfavorable. At $\mathrm{KEKB}$, some monitoring of beam lifetime is possible for

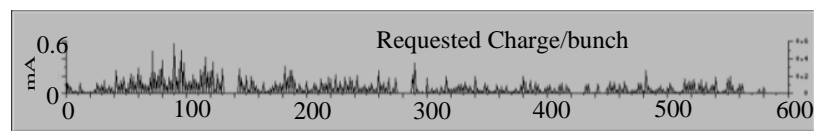

Figure 10: LER Injection requests per bunch, first 1/6 of the ring shown each ring while injection into the other ring is ongoing. At PEP-II, a "pseudo-lifetime" calculated from the decay of the charge of the bunches not recently injected is being investigated, which in principle should provide a continuous beam-lifetime measurement. Requested injection charge/time/bunch is also provided and allows the assessment of beam lifetime per bunch (Fig. 10). The example shows increased request rate for the early bunches in a train.

\section{ACKNOWLEDGMENTS}

It is a pleasure to acknowledge the work and contributions by my many colleagues of PEP-II, the BaBar detector and of KEKB and the Belle detector. For BaBar, my thanks go to C. O'Grady, M. Weaver and A. Perazzo, For PEP-II, I acknowledge J.L. Turner, M. Sullivan, F.-J. Decker, J. Seeman, M. Zisman, W. Kozanecki, A. Fisher, S. Ecklund, and for KEKB/Belle I gratefully acknowledge having received information and material from S. Uno, K. Oide, H. Koiso and Y. Funakoshi.

\section{REFERENCES}

[1] Y. Funakoshi et al., this conference.

[2] J.T. Seeman, this conference.

[3] K. Oide, private communication.

[4] PEP II, An Asymmetric B-Factory, SLAC-418, June 1993.

[5] F.-J. Decker et al., Proc. European Part. Accel. Conf., Luzern, CH, June 2004, p. 833(2004).

[6] F.-J. Decker et al., this conference.

[7] J. Olszewski et al., this conference.

[8] J. Turner et al., Proc. European Part. Accel. Conf., Luzern, CH, June 2004, p. 881.

[9] KEKB B-Factory Design Report, KEK 95-7,A, June 1995.

[10] H. Koiso, KEKB Machine Advisory Committee Presentation, Feb. 2004

[11] S. Uno, private communication. 